\title{
Derivación bilioentérica en un centro académico de referencia estatal en México
}

\author{
Bilioenteric bypass in an academic reference center in Mexico
}

\author{
Daniel Atl López Fabila, * Abel García Flores, * José Díaz Téllez,* \\ Daniel Zamora-Valdés, * Francisco Javier Nachón García*
}

Palabras clave: Derivación biliodigestiva, morbilidad, pronóstico, seguimiento.

Key words: Biliodigestive bypass, morbidity, prognosis, follow-up.

\footnotetext{
Abreviaturas:

$\mathrm{CEMEV}=$ Centro

de Especialidades

Médicas del Estado

de Veracruz "Dr.

Rafael Lucio".

$\mathrm{CTO}=$ Colangiografía

transoperatoria.

DBD $=$ Derivación

biliodigestiva.

LVB = Lesión de vía

biliar.

* Departamento de
Cirugía General,
CEMEV (Centro de
Especialidades Médicas
del Estado de Veracruz).

\section{RESUMEN}

Introducción: La selección de la derivación biliodigestiva (DBD) y una técnica quirúrgica depurada son fundamentales para la restitución del circuito enterohepático. En este trabajo se evalúan los resultados de la experiencia en DBD en el Centro de Especialidades del Estado de Veracruz "Dr. Rafael Lucio" (CEMEV). Métodos: Revisión de expedientes clínicos de pacientes sometidos a DBD en los últimos cuatro años en el CEMEV. Resultados: Se operaron 31 individuos, 27 femeninos y 4 masculinos. Las indicaciones fueron obstrucción de vía biliar $(48.39 \%)$, lesión de vía biliar (LVB) (35.48\%), síndrome de Mirizzi $(9.68 \%)$ y quiste de colédoco $(6.45 \%)$. Las DBD realizadas fueron hepaticoyeyunoanastomosis terminolateral en Y de Roux (45.16\%), coledocoduodenoanastomosis laterolateral $(19.36 \%)$, portoenteroanastomosis tipo Kasai (12.91\%), hepaticoyeyunoanastomosis laterolateral en Y de Roux con resección parcial del segmento IV $(9.68 \%)$, esfinteroplastía transduodenal $(6.45 \%)$, hepaticoyeyunoanastomosis laterolateral en omega de Braun (3.22\%) y hepaticoyeyuno en $\mathrm{Y}$ de Roux con anastomosis independientes $(3.22 \%)$. La mayor frecuencia de complicaciones se presentó en la portoenteroanastomosis $(50 \%)$ y en la coledocoduodenoanastomosis laterolateral (33.33\%). Los factores de mal pronóstico fueron LVB proximal, enfermedad biliar compleja, hemorragia intraoperatoria, colección intraabdominal, fístula biliar y sepsis. De acuerdo con los criterios de Lillemoe, obtuvimos un desenlace adecuado en el 80.65\%. Conclusión: En el CEMEV, como centro de referencia regional para patologías complejas y complicaciones hepatobiliares quirúrgicas, se requiere desarrollar una mayor experiencia en la resolución de las mismas, a fin de alcanzar los estándares internacionales. En conclusión, nuestro estudio demostró que los mejores resultados se obtuvieron con la hepaticoyeyunoanastomosis terminolateral en Y de Roux.

\section{ABSTRACT}

Background: The selection of the biliodigestive bypass and a refined surgical technique are fundamental principles in the restitution of an enterohepatic circuit. In this work, we evaluate the experience in the biliodigestive bypass surgery (DBD) in our Specialized Medical Center of Veracruz (CEMEV). Methods: A review of the medical files of the patients treated in the last four years in the CEMEV. Results: A DBD was performed in 31 cases, 27 women and four men. The indications were biliary obstruction (48.39\%), bile duct injury (LVB) (35.48\%), Mirizzie syndrome (9.68\%) and choledochal chyst (6.45\%). The DBD performed were Roux-en-Y terminolateral hepaticoyeyunostomy (45.16\%), laterolateral choledochoduodenostomy (19.36\%), Kasai portoenterostomy (12.91\%), Roux-en-Y laterolateral hepaticoyeyunostomy with partial resection of IV segment $(9.68 \%)$, transduodenal sphincteroplasty (6.45\%), Braun's omega laterolateral hepaticoyeyunostomy (3.22\%) and Roux-en-Y hepaticoyeyunostomy with independent anastomosis $(3.22 \%)$. The greater frequency of complications were found in Kasai portoenterostomy (50\%) and laterolateral choledochoduodenostomy (33.33\%). The poor prognosis factors were proximal LVB, complex biliary disease, intraoperative bleeding, intra-abdominal collection, biliary fistula and sepsis. In accordance with the criteria of Lillemoe, we obtained an adequate outcome in $80.65 \%$. Conclusions: In CEMEV, as a medical center of regional reference for complex pathologies and surgical hepatobiliary complications, it is required to develop more experience in responding to this issues, in order to meet international standards. Our experience proved that the best results were obtained with the Roux-en-Y terminolateral hepaticoyeyunostomy. 


\section{INTRODUCCIÓN}

$\mathrm{L}$ a cirugía de las vías biliares, dentro de la que se incluye la colecistectomía, es sin duda uno de los retos más complejos para el cirujano. Es común minimizar el riesgo que representa para la función y la vida; sin embargo, aun después de haber concluido una "curva de aprendizaje" adecuada, la posibilidad de una lesión de la vía biliar (LVB) es latente. Éstas se han observado con una frecuencia que oscila entre el 0.3 y el $0.6 \%$ en diferentes series en el extranjero. Un ejemplo es la serie publicada por Flum y colaboradores, auspiciada por la Asociación Médica Americana, en la que se incluyeron más de un millón y medio de pacientes, donde se reporta una incidencia de LVB del $0.5 \%$, ${ }^{1}$ ésta es similar a la reportada en México por el INNSZ y por nuestro grupo (0.4 y $0.5 \%$, respectivamente). ${ }^{2,3}$ Se han analizado estrategias con la finalidad de reducir estas cifras, tales como la realización rutinaria de colangiografía transoperatoria (CTO), sin encontrar la solución absoluta. Como ejemplo, podemos citar el metaanálisis liderado por Sheffield, en el que se incluyeron 92,932 pacientes: en el $0.21 \%$ de los casos en quienes se realizó CTO rutinaria, se produjo una LVB, y en el $0.36 \%$ de los casos en quienes no se realizó CTO rutinaria. En este estudio, sólo mediante la aplicación de pruebas estadísticas de regresión logística se pudo obtener diferencias significativas entre la realización rutinaria o selectiva de $\mathrm{CTO}$, con un OR de 1.79 [95\% Cl, 1.35-2.36]; p < 0.001, pero con pruebas habituales, la significancia de la diferencia entre la realización rutinaria y la selectiva correspondió a un OR de 1.26 [95\% Cl, 0.81-1.96]; $p=0.31 .4$ Por ello, a pesar de extremar cuidados durante los procedimientos quirúrgicos, superar las etapas de aprendizaje y la utilización de recursos tecnológicos y de imagen, las LVB continúan siendo el fantasma de las colecistectomías.

El otro gran grupo de cirugías de la vía biliar es el que se realiza como resultado de un proceso obstructivo, ya sea por litiasis, estenosis o neoplasias. La litiasis de la vía biliar se puede demostrar con CTO en el $4.6 \%$ de los pacientes sometidos a colecistectomía. ${ }^{5}$ Las estenosis biliares, menos comunes, son otro reto para el equipo multidisciplinario dedicado al manejo de la patología biliar; infortunadamente, la mayoría de las estenosis de la vía biliar son iatrógenas. ${ }^{6}$ Este tipo de lesiones pueden cursar asintomáticas por largos periodos, sin olvidar que ponen en peligro la vida, ya que con frecuencia se asocian con colangitis ascendente, absceso hepático y cirrosis biliar secundaria. ${ }^{7}$ Por otra parte, debemos considerar que un número importante de las obstrucciones de las vías biliares son secundarias a procesos malignos; un ejemplo de esto es el cáncer de páncreas como causa común de obstrucción biliar asociada con neoplasia. La mayoría de los pacientes con cáncer de páncreas mueren por extensión tumoral más que por las consecuencias de la obstrucción biliar; a pesar de ello, las obstrucciones malignas o benignas de la vía biliar pueden asociarse con síntomas alarmantes y a altos índices de morbilidad. ${ }^{8,9}$

Muchas de las lesiones quirúrgicas y los procesos obstructivos, ya sean litiásicos o estenóticos (malignos o benignos de la vía biliar), requieren de resolución quirúrgica a través de una DBD que permita la continuidad del flujo biliar, restituyendo el circuito enterohepático biliar. La reconstrucción mediante una anastomosis hepatoyeyunal en $\mathrm{Y}$ de Roux es para muchos autores el procedimiento de elección, en tanto que para otros, el uso de un asa corta yeyunal desfuncionalizada también es seguro. ${ }^{10}$ La elección del momento oportuno, la exposición completa del hilio hepático, la utilización de una anastomosis mucomucosa entre una vía biliar no fibrótica y bien vascularizada con un asa desfuncionalizada en Y de Roux sin tensión, una anastomosis amplia, la utilización de suturas absorbibles con nudos evertidos, además de la individualización en la utilización de férulas transhepáticas, parecen ser el denominador común para el éxito de la cirugía reconstructiva en la continuidad bilioentérica. ${ }^{11}$

Otros factores a considerar para la reconstrucción bilioentérica, sobre todo en lesiones complejas, son la disección parenquimatosa de Hepp-Couinad $^{12}$-que expone el conducto hepático izquierdo-, el abordaje extraglissoniano descrito por Winslow y colaboradores con anastomosis laterolateral ${ }^{13} \mathrm{y}$, finalmente, el factor de crecimiento ductal descrito por Mercado, con el que se obtiene una adecuada exposición ductal izquierda ampliando la 
boca anastomótica ductal biliar en estenosis y LVB no dilatadas. ${ }^{14}$ La portoenterostomía tipo Kasai asociada con la ferulización biliar es de suma utilidad en el manejo de lesiones complejas y en estenosis biliar no resuelta por métodos estándar. ${ }^{15}$ Los criterios de estadificación postoperatoria de Lillemoe (desenlace adecuado asintomático o sintomático sin reintervención/desenlace inadecuado asintomático o sintomático con reintervención quirúrgica o radiológica) pueden ayudar a la toma de decisiones en el manejo postoperatorio del individuo sometido a DBD. ${ }^{16}$ Por lo tanto, es importante que en cada hospital en el que se realice cirugía biliar se evalúen periódicamente las causas que condicionaron los procedimientos, las técnicas utilizadas para la resolución, el origen de su selección y los resultados a corto y largo plazo. El objetivo de esta publicación es evaluar los resultados de los casos operados de DBD realizados por el grupo de cirujanos del Centro de Especialidades Médicas del Estado de Veracruz (CEMEV) durante el periodo de junio de 2010 a junio de 2014.

\section{MATERIAL Y MÉTODOS}

Se realizó un análisis estadístico obtenido de una serie de casos por revisión de expedientes en forma retrospectiva, observacional, transversal y descriptiva. Se revisaron los expedientes de todos los pacientes mayores de 18 años sometidos a algún tipo de DBD por los integrantes del Servicio de Cirugía General del CEMEV entre el

Cuadro I. Edad, género y antecedentes de los pacientes sometidos a DBD.

Características de los pacientes sometidos a derivación biliodigestiva

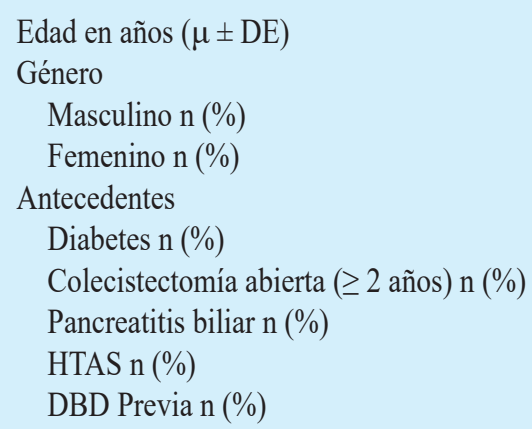

$48.6 \pm 15.6$

$$
4(12.9)
$$$$
27(87.1)
$$

1 de junio de 2010 y el 30 de junio de 2014. Se presentan tablas de frecuencia y porcentajes de la distribución por género, antecedentes, indicación de origen de la DBD, tipo de LVB de acuerdo con la clasificación de Strasberg, tipo de reconstrucción del circuito enterohepático, tasa de complicaciones inmediatas, a los seis meses y dos años, morbilidad, mortalidad y, finalmente, los criterios de estadificación postoperatoria definidos por Lillemoe.

\section{RESULTADOS}

Durante el periodo de estudio, en el CEMEV se realizaron 31 DBD en igual número de pacientes; las características demográficas y los antecedentes de importancia de los casos se presentan en el cuadro I. Se observa un franco predominio en personas de género femenino $87.09 \%$ (27) contra masculino $12.91 \%$ (4). La edad de los sujetos osciló de los 25 a los 84 años, con una media de $48.61 \pm 15.62$ (DE) años. La causa principal en esta serie que obligó a derivar la vía biliar fue la obstrucción de la misma en el $48.39 \%$ (15) de los casos. Dentro de estas obstrucciones encontramos por litiasis de la vía biliar un $32.26 \%$ (10), en los que el $60 \%$ (6) fueron por litiasis primaria (cinco en colédoco y una en los conductos hepáticos) y el 40\% (cuatro) por litiasis residual del colédoco.

Dentro de la obstrucción de la vía biliar no asociada con litiasis de la misma observamos que un $6.45 \%$ (dos) de los casos fueron derivados por obstrucción biliar de causa no identificada asociados con colangitis de repetición y un $9.68 \%$ (tres) de los casos por procesos neoplásicos. En éstos, se presentó un caso de cáncer de páncreas con intervención de primera intención curativa, y en dos casos (ampuloma duodenal y colangiocarcinoma), con fines meramente paliativos.

Las LVB correspondieron al 35.48\% (11) de los pacientes, de los cuales 10 casos se produjeron durante la colecistectomía en un 32.26\% (10) de los casos (25.81\% [ocho] abiertas contra $6.45 \%$ [dos] laparoscópicas del total de DBD), y en un $3.22 \%$ (uno), al resolver un pseudoquiste de páncreas. Cabe señalar que nuestro hospital, como centro estatal de referencia, recibió un $75 \%$ (seis) de las colecistectomías abiertas de otros nosocomios de la región. El 18.18\% (dos) 
de estas LVB fueron producidas, identificadas y tratadas inmediatamente durante la colecistectomía en nuestro nosocomio. El 3.22\% (una) de las DBD se produjo por una LVB en una intervención por un pseudoquiste pancreáti$\mathrm{CO}$, la cual fue reparada inmediatamente. En el resto de los casos de LVB $72.72 \%$ (ocho), la derivación se realizó de forma tardía ( $\geq 72$ horas). En los casos de LVB lesionados durante las colecistectomías, el $12.91 \%$ (cuatro) nos fueron referidos con lesiones agudas tratadas con DBD previa en sus hospitales de origen, las cuales presentaron estenosis temprana ( $\leq$ tres años). Éstas fueron tres coledocoduodenoanastomosis laterolateral remodeladas, dos a hepaticoyeyunoanastomosis terminolateral en $Y$ de Roux y una a hepaticoyeyunoanastomosis

\section{Cuadro II. Causas de la derivación biliodigestiva.}

\begin{tabular}{lrc} 
Causas de la derivación biliodigestiva & Núm. & (\%) \\
\hline Obstrucción de la vía biliar & 15 & 48.3 \\
Litiasis primaria de colédoco & 5 & 16.1 \\
Litiasis residual del colédoco & 4 & 12.9 \\
Litiasis primaria de conductos hepáticos & 1 & 3.2 \\
Neoplasias de V.B. o páncreas & 3 & 9.7 \\
Estenosis de causa no identificada & 2 & 6.4 \\
Lesiones de la vía biliar & 11 & 35.4 \\
Colecistectomía & 10 & 32.2 \\
Tratamiento de pseudoquiste de páncreas & 1 & 3.2 \\
Síndrome de Mirrizi & 3 & 9.7 \\
Quiste de colédoco & 2 & 6.4 \\
Total & 31 & 100
\end{tabular}

\begin{tabular}{|c|c|c|}
\hline \multicolumn{3}{|c|}{$\begin{array}{l}\text { Cuadro III. Distribución de las lesiones } \\
\text { de la vía biliar de acuerdo } \\
\text { con la clasificación de Strasberg. }\end{array}$} \\
\hline Tipo & (n) & $\%$ \\
\hline E1 & 3 & 27.2 \\
\hline E2 & 2 & 18.1 \\
\hline E3 & 4 & 36.3 \\
\hline E4 & 1 & 9.1 \\
\hline E5 & 1 & 9.1 \\
\hline Total & 11 & 100 \\
\hline
\end{tabular}

laterolateral en Y de Roux con resección parcial del segmento IV. La última fue una hepaticoyeyunoanastomosis terminolateral en $\mathrm{Y}$ de Roux que se remodeló a una portoenterostomía tipo Kasai. Esta última presentó una fístula biliar manejada por instrumentación radiológica resuelta al año de la cirugía y una estenosis al segundo año tratada de la misma forma, por medio de stents transhepáticos periódicos con resolución desfavorable y evolución clínica hacia la cirrosis hepática secundaria. Las demás DBD remodeladas mencionadas presentaron un desenlace adecuado de acuerdo con los criterios de Lillemoe.

Un 9.68\% (tres) de los casos fueron sometidos a DBD por fístula colecistocoledociana (síndrome de Mirizzi), con disrupción de más del $60 \%$ de la circunferencia del colédoco de estos casos. En el $6.45 \%$ (dos) se realizaron DBD por quistes de colédoco tipo I de acuerdo con la clasificación de Todani (Cuadro II). En relación con los tipos de LVB, se dispusieron de acuerdo con la clasificación propuesta por Strasberg. ${ }^{17}$ Prácticamente todas las LVB fueron englobadas en lesiones tipo E; de éstas, los subtipos más frecuentes fueron E3 en un $36.37 \%$ (cuatro) de los casos, E1 en un $27.27 \%$ (tres), E2 en un $18.18 \%$ (dos), E4 y E5 en un $9.09 \%$ (uno) cada uno (Cuadro III).

Para el establecimiento de la continuidad del flujo biliar, se utilizaron diferentes tipos de anastomosis en diferentes segmentos intestinales, utilizando un asa desfuncionalizada en Y de Roux en el 70.97\% (22) de los casos. En el $25.81 \%$ (ocho) de los casos, la derivación se realizó en duodeno, y en el 3.22\% (uno), se utilizó el yeyuno a través de un asa en omega de Braun. Las DBD más utilizadas en forma descendente fueron hepaticoyeyunoanastomosis terminolateral en $\mathrm{Y}$ de Roux en el $45.16 \%$ (14), coledocoduodenoanastomosis laterolateral en el $19.36 \%$ (seis), portoenterostomía tipo Kasai en el $12.91 \%$ (cuatro), hepaticoyeyunoanastomosis laterolateral en $\mathrm{Y}$ de Roux con resección parcial del segmento IV en un $9.68 \%$ (3), esfinteroplastía transduodenal en el $6.45 \%$ (dos), hepaticoyeyunoanastomosis laterolateral en omega de Braun en el 3.22\% (uno) y hepaticoyeyuno en $\mathrm{Y}$ de Roux con anastomosis independientes en el 3.22\% (uno) (Cuadro IV). En el 3.22\% (uno) de los casos, 
Cuadro IV. Tipos de derivaciones biliodigestivas empleadas.

Tipo de derivación biliodigestiva (técnica quirúrgica)

Hepaticoyeyunoanastomosis terminolateral*

Coledocoduodeno anastomosis laterolateral

Portoenteroanastomosis tipo Kasai*

Hepaticoyeyunoanastomosis laterolateral en $\mathrm{Y}$ de Roux con resección parcial del

segmento IV*

Esfinteroplastía transduodenal

Hepaticoyeyunoanastomosis laterolateral en omega de Braun

Hepaticoyeyuno con anastomosis independientes**

Total

\section{(n)}

$\begin{array}{rr}14 & 45.1 \\ 6 & 19.3 \\ 4 & 12.9 \\ 3 & 9.6\end{array}$

$\begin{array}{rc}2 & 6.4 \\ 1 & 3.2 \\ 1 & 3.2 \\ 31 & 100\end{array}$

* La derivación se realizó utilizando un asa desfuncionalizada en Y de Roux.

** Se realizó con segmentectomía hepática del IV y V.

debido a una LVB Strasberg E5 (lesión de un conducto derecho posterior aberrante junto con sección de la confluencia ductal hepática), se optó por realizar DBD con anastomosis independientes con abordaje de Hepp-Couinad junto con segmentectomías hepáticas IV y V, con anastomosis laterolateral mucomucosa ductal izquierda y portoenterostomía tipo Kasai mucocapsular ductal derecha con ferulización transhepática derecha, la cual evolucionó a fístula biliar remitida a los seis meses con apoyo de instrumentación radiológica.

Por las características del sistema de referencia y contrarreferencia del CEMEV, no pudo ser posible establecer un seguimiento a largo plazo de los pacientes; sin embargo, logramos evidenciar la evolución clínica en el expediente en la mayoría de los individuos, en un $93.54 \%$ (29), hasta los seis meses del postoperatorio, y en un $64.51 \%$ (20) de los casos a los dos años. Aunque la mayoría de los sujetos evolucionaron satisfactoriamente, se detectaron complicaciones inmediatas en el 61.29\% (19) del total, la mayoría resueltas favorablemente. Se identificaron lesiones vasculares asociadas en el $12.91 \%$ (cuatro) (arteria hepática o lesión portal) dentro de la hemorragia transoperatoria presentada en el $22.58 \%$ (siete) de los casos. Éstas ameritaron en dos casos resolución por portoenterostomía tipo Kasai, y en los otros dos por hepaticoyeyunoanastomosis laterolateral en Y de Roux con resección parcial del segmento
IV. Los días de EIH fueron en promedio de 20.59 días (DE 16.81/4-70). Se presentaron fístulas biliares en un $25.81 \%$ (ocho) de los casos, de las cuales cinco resolvieron con manejo conservador sin necesidad de reintervención.

Durante los seis meses de seguimiento y antes de su referencia definitiva a sus centros de adscripción, se detectaron complicaciones en un $9.68 \%$ (tres) de los casos. En un 6.45\% (dos) de los casos (una coledocoduodenoanastomosis laterolateral y una hepaticoyeyunoanastomosis laterolateral en $\mathrm{Y}$ de Roux con anastomosis independientes), se desarrollaron fístulas biliares; la última ameritó reintervención radiológica para su resolución. En el 3.2\% (uno), una coledocoduodenoanastomosis laterolateral desarrolló estenosis manejada por intervención radiológica con drenaje transhepático, con una evolución favorable. En el seguimiento a dos años, se encontraron complicaciones en el $9.7 \%$ (tres) de los casos. Se observó en el $3.2 \%$ (uno) un caso de portoenterostomía tipo Kasai que inició con fístula biliar y evolucionó a estenosis y, posteriormente, a cirrosis hepática, el cual ameritó reintervención radiológica con manejo paliativo multidisciplinario. En el 6.45\% (dos) de los casos se presentaron estenosis de la DBD; una de éstas de una estenosis previa ya descrita a los seis meses de seguimiento (una coledocoduodenoanastomosis terminolateral) y una nueva portoenterostomía tipo Kasai, las cuales fueron tratadas por medio de instrumen- 
tación radiológica, con un desenlace adecuado. Se presentó una tasa de mortalidad perioperatoria del $6.45 \%$ (dos): en una coledocoduodenoanastomosis laterolateral por choque séptico y en una hepaticoyeyunoanastomosis laterolateral con resección parcial del segmento IV en Y de Roux por choque hipovolémico y séptico (Cuadro V).

En el análisis global, de acuerdo con los criterios de estadificación postoperatoria utilizados por Lillemoe, encontramos un desenlace adecuado en el $77.42 \%$ (24) de los casos, los cuales no ameritaron reintervención. Sin embargo, el $22.58 \%$ (siete) restantes ameritaron algún tipo de reintervención (radiológica o quirúrgica) o presentaron mortalidad. Se observa que la proporción por tipo de DBD no fue equitativa en su totalidad. Tomando en cuenta la variabilidad del tipo de DBD, se presentó, en orden descendente, un desenlace adecuado en la hepaticoyeyunostomía en omega de Braun $100 \%$ (uno), la esfinteroplastía transduodenal $100 \%$ (dos), la hepaticoyeyunostomía terminolateral en Y de Roux 100\% (14), y la hepa- toyeyunostomía laterolateral con resección del segmento IV en Y de Roux $66.67 \%$ (dos), como se observa en el cuadro VI.

\section{DISCUSIÓN}

El desenlace satisfactorio en la DBD continúa siendo un reto para muchos cirujanos en el contexto de la morbilidad asociada con el padecimiento actual que dirige el procedimiento. Limitaciones importantes de nuestro estudio son su carácter retrospectivo, la muestra poblacional y la variabilidad del padecimiento, así como del tipo de DBD que conllevó su resolución. Al comparar otras series más grandes de DBD, como la de Altaf y colaboradores, y la nuestra observamos mayor prevalencia de LVB (35.48\% contra $25.21 \%$ ) y obstrucción de vía biliar asociada con litiasis $(32.26 \%$ contra $25.32 \%$ ) en nuestra serie. Esto refleja el peso de la LVB y la coledocolitiasis en las DBD en nuestra serie. Aunque cabe señalar que la mayoría de las LVB se dieron en colecistectomías abiertas frente a las laparoscópicas, lo cual

Cuadro V. Evolución de los pacientes sometidos a derivación biliodigestiva; se muestran las complicaciones inmediatas, las complicaciones a los seis meses, a los dos años de seguimiento y la mortalidad.

Complicaciones y mortalidad

\begin{tabular}{|c|c|c|c|c|c|c|c|c|c|c|c|}
\hline $\begin{array}{l}\text { Complicaciones } \\
\text { inmediatas }\end{array}$ & (n) & $\%$ & A los 6 meses & (n) & $\%$ & $A$ los 2 años & (n) & $\%$ & Mortalidad & (n) & $\%$ \\
\hline Fístula biliar & 8 & 25.8 & Fístula biliar & 2 & 6.5 & Cirrosis & 1 & 3.2 & Sepsis & 2 & 6.5 \\
\hline Hemorragia & 7 & 22.6 & $\begin{array}{l}\text { Estenosis } \\
\text { de DBD }\end{array}$ & 1 & 3.2 & $\begin{array}{l}\text { Estenosis } \\
\text { de DBD }\end{array}$ & 3 & 9.7 & & & \\
\hline $\begin{array}{l}\text { Lesión vascular } \\
\text { identificada }\end{array}$ & 4 & 12.9 & $\begin{array}{l}\text { Colección } \\
\text { postquirúrgica }\end{array}$ & 1 & 3.2 & & & & & & \\
\hline $\begin{array}{l}\text { Colecciones } \\
\text { postquirúrgicas }\end{array}$ & 5 & 16.1 & & & & & & & & & \\
\hline $\begin{array}{l}\text { Infección de sitio } \\
\text { quirúrgico }\end{array}$ & 5 & 16.1 & & & & & & & & & \\
\hline Colangitis & 4 & 12.9 & & & & & & & & & \\
\hline $\begin{array}{l}\text { Dehiscencia } \\
\text { anastomosis }\end{array}$ & 2 & 6.5 & & & & & & & & & \\
\hline Biliopatía portal & 1 & 3.2 & & & & & & & & & \\
\hline Absceso abdominal & 1 & 3.2 & & & & & & & & & \\
\hline Fístula yeyunal & 1 & 3.2 & & & & & & & & & \\
\hline Fístula pancreática & 1 & 3.2 & & & & & & & & & \\
\hline
\end{tabular}




\begin{tabular}{|c|c|c|c|c|}
\hline \multicolumn{5}{|c|}{$\begin{array}{c}\text { Cuadro VI. Evolución de los pacientes sometidos a derivación biliodigestiva de acuerdo } \\
\text { con los criterios de estadificación postoperatoria de Lillimoe (desenlace adecuado asintomático } \\
\text { o sintomático sin reintervención/desenlace inadecuado asintomático o sintomático } \\
\text { con reintervención quirúrgica o radiológica). }\end{array}$} \\
\hline \multicolumn{5}{|c|}{ Pronóstico } \\
\hline \multirow[b]{2}{*}{ Tipo de derivación biliodigestiva } & \multicolumn{2}{|c|}{ Desenlace adecuado } & \multicolumn{2}{|c|}{ Desenlace inadecuado } \\
\hline & (n) & $\%$ & (n) & $\%$ \\
\hline Hepaticoyeyunoanastomosis terminolateral & 14 & 100 & -- & -- \\
\hline Coledocoduodenoanastomosis laterolateral & 4 & 66.7 & 2 & 33.3 \\
\hline Portoenteroanastomosis tipo Kasai & 2 & 50 & 2 & 50 \\
\hline $\begin{array}{l}\text { Hepaticoyeyunoanastomosis laterolateral en Y de } \\
\text { Roux con resección parcial del segmento IV }\end{array}$ & 2 & 66.7 & 1 & 33.3 \\
\hline Esfinteroplastía transduodenal & 2 & 100 & -- & -- \\
\hline $\begin{array}{l}\text { Hepaticoyeyunoanastomosis laterolateral en } \\
\text { omega de Braun }\end{array}$ & 1 & 100 & -- & -- \\
\hline Hepaticoyeyuno con anastomosis independientes & -- & -- & 1 & 100 \\
\hline Total & 25 & 80.7 & 6 & 19.3 \\
\hline
\end{tabular}

pudiera reflejar una curva de aprendizaje que debe ser reforzada en el procedimiento abierto debido al incremento de la cirugía laparoscópica, junto con la complejidad de la patología de fondo, ya que de estas LVB, el 75\% fueron referidas de otros nosocomios. ${ }^{18} \mathrm{La}$ frecuencia de LVB observada fue similar a otras series, E1E3, 81.82\% (nueve) y E4-E5, 18.8\% (dos), como la de Mercado, con una frecuencia E1-E3 del 78\% (109) y E4-E5 del 22\% (31). ${ }^{19}$ Los factores determinantes de elección observados en las DBD que ameritaron el abordaje por segmentectomía en el $12.91 \%$ (cuatro) del total que se hicieron fueron la lesión vascular asociada en el 50\% (dos), la LVB Strasberg E4 50\% (dos) y la DBD previa que se presentó junto con lesión vascular en un 25\% (uno). Esta observación es similar a los hallazgos reportados en la revisión de Truant y colaboradores, donde informan la lesión arterial hepática y la LVB Strasberg E4 y E5 como factores independientes asociados a hepatectomías. ${ }^{20}$ Cabe señalar que estos factores se pueden presentar juntos en una misma patología y DBD, lo que modifica notablemente la evolución postquirúrgica.

En el tipo de DBD realizadas concordamos con la mayoría de los autores en que la hepatoyeyunostomía en $Y$ de Roux es la de elección en casi todas las patologías que ameritan resolución por una derivación. La coledocoduodenostomía es una opción que debe ser usada con precaución. En algunos casos, el duodeno se encuentra libre de inflamación y su adecuada movilización puede asegurar una anastomosis libre de tensión. La tasa de fugas de esta anastomosis puede ser menor, aunque en nuestra serie esto no se refleja en el seguimiento por la mayor prevalencia de fístulas biliares. Sin embargo, la presentación de las mismas puede ser la de perforación intestinal, en comparación con la Y de Roux, en la que la fuga biliar y el asa está desfuncionalizada. La remodelación en las DBD estenosadas previamente realizada en la presente serie demuestra que, de acuerdo con las circunstancias patológicas intrínsecas y técnicas, se debe intentar garantizar una aproximación lo más hermética posible, ya sea con una hepaticoyeyunoanastomosis terminolateral o laterolateral, con o sin disección de la placa hiliar y/o resección segmentaria en la medida de lo posible, ya que esto técnicamente podría garantizar una aproximación mucomucosa. Observamos que al asegurar una aproximación mucomucosa, el índice de fístula biliar 
disminuía; por tanto, se optó en algunos casos por realizar algún tipo de segmentectomía. Con esto, creemos que este abordaje es útil e importante en el armamento quirúrgico para garantizar una aproximación mucomucosa, tomando siempre en consideración la experiencia quirúrgica. ${ }^{21}$ Un ejemplo de este principio se observó cuando se tuvo una LVB Strasberg E5, donde se realizó una hepaticoyeyunoanastomosis en $Y$ de Roux laterolateral ductal izquierda con aproximación mucomucosa junto con una portoenterostomía mucocapsular ductal derecha con ferulización transhepática debido a las características propias descritas de la lesión, donde sólo se tuvo que reintervenir radiológicamente por una fístula biliar, la cual remitió favorablemente (Figura 1).

Infortunadamente, no fue posible valorar con exactitud el tiempo de referencia, así como el de reparación de la DBD a partir del diagnósti$\mathrm{Co}$, lo cual puede reflejar parte de nuestros resultados. En nuestra experiencia, se tuvo estenosis de las DBD (una coledocoduodenoanastomosis y dos portoenterostomías tipo Kasai) en el 9.68\% (tres), estas mismas asociadas con fístulas biliares y hemorragia transoperatoria, lo que enfatiza el esfuerzo por una adecuada selección de la técnica y la mejora de la misma en la búsqueda de un desempeño quirúrgico satisfactorio.

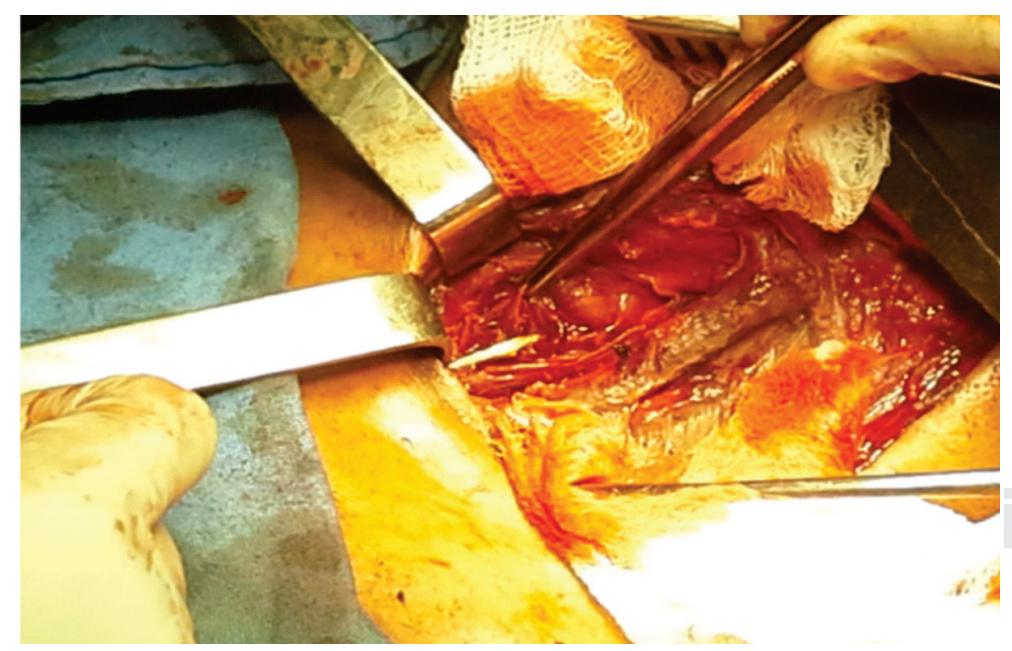

Figura 1. Lesión de vía biliar Strasberg E5 con conducto hepático derecho posterior aberrante, exploración del conducto hepático derecho. Reconstrucción con hepaticoyeyuno más segmentectomía del IV y V con anastomosis independientes mucomucosa izquierda y mucocapsular derecha tipo Kasai con stent transhepático derecho.
Con esto, se observa que los factores pronósticos de pobre evolución postoperatoria fueron LVB proximal, enfermedad biliar compleja, hemorragia intraoperatoria, colección intraabdominal, fístula biliar y sepsis. De acuerdo con la estadificación postoperatoria, se observó un desempeño satisfactorio en un $80.65 \%$ (25). Este desempeño requiere ser desarrollado en criterio clínico-quirúrgico para lograr acercarse a algunas grandes series como la de Mercado, donde se alcanza un desenlace satisfactorio del $91 \% .^{22}$ Creemos que tanto la variabilidad en el tipo de DBD como la causa de la misma, la selección, el desarrollo de la técnica y nuestra experiencia reflejan estos resultados. Enfatizamos el mayor seguimiento, limitado en esta serie, la selección de la técnica quirúrgica, así como el desarrollo y desempeño quirúrgico adecuado en la misma con el fin de garantizar la permeabilidad de una DBD a largo plazo.

\section{CONCLUSIÓN}

Existe camino por recorrer en la curva de aprendizaje en una búsqueda incesante de la excelencia quirúrgica como centro de referencia a nivel estatal. La hepaticoyeyunoanastomosis terminolateral en $\mathrm{Y}$ de Roux es el procedimiento de elección en nuestra experiencia, con el menor índice de morbilidad a corto y largo plazo. Se debe optar por garantizar una anastomosis mucomucosa amplia sin tensión, si es necesario con segmentectomía hepática, de acuerdo con la experiencia quirúrgica. En caso contrario, debido a la naturaleza de la patología, la portoenterostomía tipo Kasai con ferulización transhepática es la mejor opción. Por consiguiente, este tipo de técnicas quirúrgicas tendrán que ser ejercitadas, desarrolladas y promovidas en el armamento quirúrgico del cirujano. Por ende, el diagnóstico, la referencia oportuna, la buena selección y desarrollo de la técnica quirúrgica, junto con un adecuado cuidado y seguimiento postquirúrgico, determinarán el desenlace satisfactorio de una adecuada DBD. El presente trabajo es un esfuerzo por mejorar la evaluación y evolución de los aspectos médico-quirúrgicos en el equipo multidisciplinario responsable de mejorar el desempeño quirúrgico y el éxito de la derivación biliodigestiva. 


\section{Agradecimientos}

Al maestro Hidromiro Florentino Murrieta Guevara, por su ejemplo y esfuerzo en nuestra formación médica y humana.

\section{CONFLICTO DE INTERESES}

Los autores declaran no tener ningún conflicto de intereses.

\section{REFERENCIAS}

1. Flum DR, Cheadle A, Prela C, Dellinger EP, Chan L. Bile duct injury during cholecystectomy and survival in medicare beneficiaries. JAMA. 2003; 290: 21682173.

2. Mercado MA, Chan C, Tielve M, Contreras A, GálvezTreviño R, Ramos-Gallardo G, et al. Lesión iatrógena de la vía biliar. Experiencia en la reconstrucción en 180 pacientes. Rev Gastroenterol Mex. 2002; 67: 245-249.

3. Nachón-García FJ, Díaz-Téllez J, Martín-Bendímez J, Murrieta-Guevara H, Namihira-Guerrero D. Colecistectomía laparoscópica. Experiencia de cinco años en el Centro de Especialidades Médicas del Estado de Veracruz. Cir Cirj. 2001; 69: 22-25.

4. Sheffield KM, Riall TS, Han Y, Kuo YF, Townsend CM Jr, Goodwin JS. Association between cholecystectomy with vs without intraoperative cholangiography and risk of common duct injury. JAMA. 2013; 310: 812-820.

5. Collins C, Maguire D, Ireland A, Fitzgerald E, O'Sullivan GC. A prospective study of common bile duct calculi in patients undergoing laparoscopic cholecystectomy: natural history of choledocholithiasis revisited. Ann Surg. 2004; 239: 28-33.

6. Vecchio R, Ferrara M, Pucci L, Meli G, Latteri S. Treatment of iatrogenic lesions of the common bile duct. Minerva Chir. 1995; 50: 29-38.

7. Hanau LH, Steigbigel NH. Acute (ascending) cholangitis. Infect Dis Clin North Am. 2000; 14: 521-546.

8. Hastier P, Buckley JM, Peten EP, Dumas R, Delmont J. Long-term treatment of biliary stricture due to chronic pancreatitis with a metallic stent. Am J Gastroenterol. 1999; 94: 1947-1948.

9. Magistrelli P, Masetti R, Coppola R, Riccioni ME, Crucitti A, Nuzzo G. Changing attitudes in the palliation of proximal malignant biliary obstruction. J Surg Oncol Suppl. 1993; 3: 151-153.

10. Felder SI, Menon VG, Nissen NN, Margulies DR, Lo S, Colquhoun S. Hepaticojejunostomy using short-limb Roux-en-Y reconstruction. JAMA Surg. 2013; 148: 253-258.
11. Mercado MA. Iatrogenia de vías biliares. Capitulo 13. Técnicas de reconstrucción. México: Editorial Corinter; 2010. pp. 182-190.

12. Myrburgh AJ. The Hepp-Couinad approach to strictures of the bile ducts. Ann Surg. 1993; 218: 615-620.

13. Winslow RE, Fialkowski AE, Linehan DC, Hawkins GW, Picus DD, Strasberg MS. "Sideways": results of repair of biliary injuries using a policy of side-to-side hepatico-jejunostomy. Ann Surg. 2009; 249: 426-434.

14. Mercado MA, Orozco H, Chan C, Quezada C, BarajasOlivas A, Borja-Cacho D, et al. Bile duct growing factor: an alternate technique for reconstruction of thin bile ducts after iatrogenic injury. J Gastrointest Surg. 2006; 10: 1164-1169.

15. Gao JB, Bai LS, Hu ZJ, Wu JW, Chai XQ. Role of Kasai procedure in surgery of hilar bile duct strictures. World J Gastroenterol. 2011; 17: 4231-4234.

16. Lillemoe KD, Melton GB, Cameron JL, et al. Postoperative bile strictures: management and outcome in the 1990s. Ann Surg. 2000; 232: 430-431.

17. Strasberg SM, Hertl M, Soper NJ. An analysis of the problem of biliary injury during laparoscopic cholecystectomy. J Am Coll Surg. 1995; 180: 101-125.

18. Altaf HK, Arshad MM, Amir IM, Jawed NQ, Ahmed KS, Abdul AL. Biliary bypass surgery-analysis of indications \& outcome of different procedures. Pak J Med Sci. 2013; 29: 799-802.

19. Mercado MÁ, Franssen B, Dominguez I, ArriolaCabrera JC, Ramírez-Del Val F, Elnecavé-Olaiz A, et al. Transition from a low-to a high-volume center for bile duct repair: changes in technique and improved outcome. HPB (Oxford). 2011; 13: 767-773.

20. Truant S, Emmanuel-Boleslawski E, Lebuffe G, Géraldine-Sergent G, Pruvot FR. Hepatic resection for post-cholecystectomy bile duct injuries: a literature review. HPB (Oxford). 2010; 12: 334-341.

21. Mercado MA, Chan C, Orozco H, Villalta MJ, Barajas OA, Eraña J. Long-term evaluation of biliary reconstruction after partial resection of segments IV and $\mathrm{V}$ in iatrogenic injuries. J Gastrointest Surg. 2006; 10: 77-82.

22. Mercado MA, Orozco H, De la Garza L, LópezMartínez ML, Contreras A, Guillén-Navarro E. Biliar duct injury. Partial segment IV resection for intrahepatic reconstruction of biliary injuries. Arch Surg. 1999; 134: 1008-1010.

Correspondencia:

\section{Daniel Atl López Fabila}

Calle Ayuntamiento Núm. 26 (Interior

\# 33-Fraccionamiento Villas Tabachines),

Colonia Centro, 76800,

San Juan del Río, Querétaro.

Teléfono: 4272725359

Celular: 2281711491

E-mail: jackatl7@hotmail.com 